\title{
High-vacuum-compatible high-power Faraday isolators for gravitational-wave interferometers
}

\author{
Oleg V. Palashov, ${ }^{1}$ Dmitry S. Zheleznov, ${ }^{1, *}$ Alexander V. Voitovich, ${ }^{1}$ Victor V. Zelenogorsky, ${ }^{1}$ \\ Eugene E. Kamenetsky, ${ }^{2}$ Efim A. Khazanov, ${ }^{1}$ Rodica M. Martin, ${ }^{3}$ Katherine L. Dooley, ${ }^{3}$ Luke Williams, ${ }^{3}$ \\ Antonio Lucianetti, ${ }^{4}$ Volker Quetschke, ${ }^{5}$ Guido Mueller, ${ }^{3}$ David H. Reitze, ${ }^{3,6}$ David B. Tanner, ${ }^{3}$ \\ Eric Genin, ${ }^{7}$ Benjamin Canuel, ${ }^{7}$ and Julien Marque ${ }^{7}$ \\ ${ }^{1}$ Institute of Applied Physics of the Russian Academy of Sciences, 46 Ulyanova Street, Nizhny Novgorod, 603950, Russia \\ ${ }^{2}$ Department of Physics, New York University, New York, New York 10003, USA \\ ${ }^{3}$ Department of Physics, University of Florida, Gainesville, Florida 32611, USA \\ ${ }^{4}$ HiLASE Project, Institute of Physics ASCR v.v.i., Na Slovance 2, 18221 Prague, Czech Republic \\ ${ }^{5}$ Department of Physics and Astronomy, University of Texas at Brownsville, Brownsville, Texas 78520, USA \\ ${ }^{6}$ LIGO Laboratory, California Institute of Technology, MS 100-36, Pasadena, California 91125, USA \\ ${ }^{7}$ European Gravitational Observatory (EGO), I-56021 Cascina, Pisa, Italy \\ ${ }^{*}$ Corresponding author: zheleznov@gmail.com
}

Received January 19, 2012; revised April 6, 2012; accepted April 17, 2012;

posted April 18, 2012 (Doc. ID 161872); published June 27, 2012

Faraday isolators play a key role in the operation of large-scale gravitational-wave detectors. Second-generation gravitational-wave interferometers such as the Advanced Laser Interferometer Gravitational-Wave Observatory (LIGO) and Advanced Virgo will use high-average-power cw lasers (up to $200 \mathrm{~W}$ ) requiring specially designed Faraday isolators that are immune to the effects resulting from the laser beam absorption-degraded isolation ratio, thermal lensing, and thermally induced beam steering. In this paper, we present a comprehensive study of Faraday isolators designed specifically for high-performance operation in high-power gravitational-wave interferometers. (C) 2012 Optical Society of America

OCIS codes: $\quad 230.2240,140.6810,230.3240,230.3810$.

\section{INTRODUCTION}

Faraday isolators (FIs) are optical devices that are widely used in applications where isolation from backpropagating laser light is required or desirable. Applications involving high-average-power lasers and operation in a vacuum environment place extra demands on FI performance. One such application is gravitational-wave interferometry. In all advanced gravitational-wave interferometers [1-5], any noise introduced by backreflected and parasitic beams can compromise the sensitivity of an interferometer through the introduction of spurious phase noise. In particular, the frequency stability of the input beam to the interferometer is affected by the main back reflection of the interferometer itself towards the injection system. FIs are placed on the external (in-air) laser as well as on in-vacuum optical benches in order to prevent light reflected by the interferometer and input optics components from propagating back into the laser.

FIs used in future gravitational wave interferometers must satisfy two overarching requirements in addition to producing high isolation ratios. First, they must work with high average laser powers (up to $185 \mathrm{~W}$ for Advanced LIGO [5] , $200 \mathrm{~W}$ for Advanced Virgo [2], and $150 \mathrm{~W}$ for the Large Cryogenic Gravitational-Wave Telescope [6]). This places extra demands on minimizing thermal effects that can compromise the laser beam quality and reduce the optical isolation ratio. Second, they must operate in a high-vacuum environment. These requirements are coupled to each other-in the absence of convective cooling of the magneto-optical elements (MOEs), thermally induced effects due to the absorption of the laser beam should be minimized in high-power FIs using alternative methods. (A complete list of requirements for the Advanced LIGO FI can be found in [7].)

The paper describes the concept and characteristics of magnetic systems (MS) and optical elements, the design and assembly features of the vacuum compatible high-power FIs (VPFIs), installed inside the LIGO and Virgo interferometers. As we describe below, both the LIGO and Virgo designs are similar in concept and share similar design elements but are different in detailed implementation. Dependences of the isolation ratio on pressure, the thermal lens in MOE on the laser power, and beam thermal drift versus time are given. As a conclusion, the near-term prospects of development of the VPFI for laser radiation with average power of subkilowatt levels are discussed. In Section 2, we describe the design of the VPFI, including modifications that enable in-vacuum compatible operation, the magnet system and field configuration, and high thermal conductivity holders for the MOEs. Section 3 presents the VPFI performance characterization for both the LIGO and Virgo versions, including the dependence of the isolation ratio on input power and pressure, thermal lensing versus power, and thermal beam steering. We conclude in Section 4.

\section{DESIGN OF HIGH-VACUUM-COMPATIBLE HIGH-POWER FARADAY ISOLATOR}

We approach the VPFI design in multiple ways. The use of thermal birefringence compensation [ $\underline{8}]$ and thermal lens compensation techniques [9,10] allows for significantly greater laser powers over conventional FI designs. Selection 
of low-absorption optical elements [terbium gallium garnet (TGG) magneto-optical crystals and polarizers] minimizes the amount of absorbed power and subsequent thermal gradients that lead to thermal lensing and depolarization. The design of compact, ultrahigh field magnets for the Faraday rotator permits the use of shorter length magneto-optical crystals that further minimize thermal loading. To improve heat conduction in the vacuum environment, specially designed sleeves are used to mount the TGG crystals. Finally, we use a motorized half-wave plate in-vacuum to allow for remote adjustment of the isolation ratio.

\section{A. Vacuum Design}

Gravitational-wave interferometers operate in ultrahigh vacuum to reduce phase noise introduced from light scattering of residual gas. (LIGO operates at $10^{-9}$ torr, Virgo operates at $7.5 \cdot 10^{-7}$ torr.) Both the Advanced LIGO and Advanced Virgo VPFIs use a modular vacuum-compatible design that meets the stringent requirements for low outgassing [11].

The Faraday rotator housing contains the two TGG crystals (20 mm diameter, $10 \mathrm{~mm}$ length, [001] orientation) and one quartz crystal (SPECS) and the magnetic rings that provide the axial field in the area where the crystals are situated. The MS of the VPFI consists of seven magnetic annuli composed of Nd-Fe-B permanent magnets, of which six have a radially directed magnetization vector: three rings radially directed toward center, three rings directed away from the center, and one solid ring with the magnetization vector directed on axis [12]. The axial and radial repulsive forces between the disks are substantial, approximately 70,000 (36,000) psi in the axial (radial) direction [13]. Thus the case is a single aluminum "cup" with $9 \mathrm{~mm}$ wall thickness and one end cap to contain the magnets. The case is slotted to vent the FR during vacuum bake-out (Fig. 1). Shims are used both between the magnetic disks [Fig. 2(b), $0.5 \mathrm{~mm}$ thick] and within the eight magnets comprising each disk [Fig. 2(a), $1 \mathrm{~mm}$ thick] to facilitate venting during the bakeout. Shim thicknesses were chosen based on an analysis that computed the minimum required for efficient vacuum venting $(0.3 \mathrm{~mm})$ and the maximum limit that could be used without reducing the magnitude and homogeneity of the magnetic field $(1 \mathrm{~mm})$.
Vacuum baking is required to eliminate contaminants from the $\mathrm{Nd}-\mathrm{Fe}-\mathrm{B}$ magnets during fabrication and processing. These high coercivity Nd-Fe-B magnets have low Curie temperatures [14], and thus cannot be subjected to high temperatures. The magnetic rings were baked for a $48 \mathrm{~h}$ duration at $60{ }^{\circ} \mathrm{C}$ and monitored for outgassing. To verify that the sustained high temperatures did not induce demagnetization, the magnets were measured before and after baking. The dependence of the longitudinal component of one ring magnetic field, measured before and after the baking, is shown in Fig. 3 . The fields are identical, indicating that the magnetic field does not diminish during the baking process.

\section{B. Magnetic Field Distribution}

For light traversing an MOE in the Faraday rotator, the polarization rotation angle is given by

$$
\varphi(r)=V(r) \int_{0}^{L_{\mathrm{MOE}}} B_{z}(z, r) \mathrm{d} z,
$$

where $\varphi$ is the linear polarization rotation angle, $V$ is the Verdet constant, $B_{z}$ is the axial magnetic field, and $L_{\mathrm{MOE}}$ is the length of MOE. The radial dependence comes about from the photoelastic effect and the temperature dependence of the Verdet constant, both driven by the laser field, as well as from magnetic field inhomogeneity. These effects have been investigated extensively by some of the present authors and others [15-19], and it was found that the photoelastic effect is the dominant reason for the radially dependent polarization appearance. To minimize radially dependent polarization, reduced magneto-optic crystal lengths are needed. It is clear from Eq. (1) that it could be provided by large magnetic fields in VPFIs.

The VPFIs are located in close proximity to suspended optical mirrors that have small permanent magnets mounted to them to allow magnetic actuation for position and angular control [20]. Magnetic fields generate forces and torques on the magnets given by $\vec{F}=\vec{\nabla}(\vec{\mu} \bullet \vec{B})$ and $\vec{\tau}=\vec{\mu} \times \vec{B}$ where $\vec{\mu}$ is the magnetic dipole moment of the magnet. These forces and torques, if sufficiently large, present a problem when $\vec{B}$

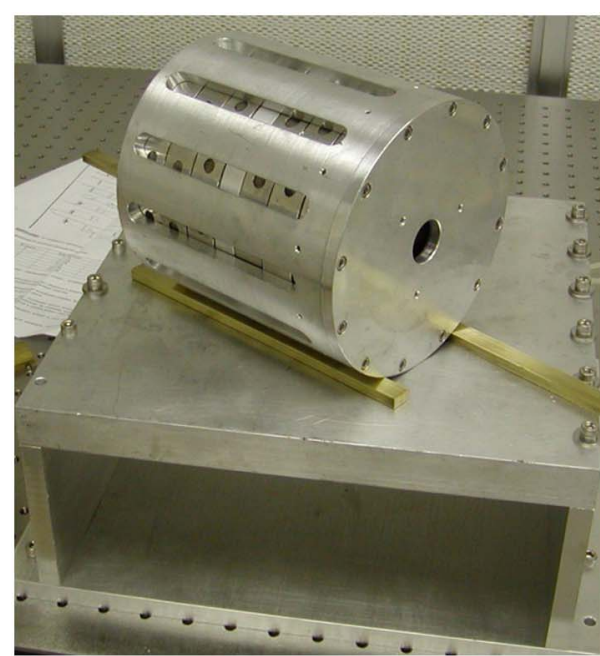

(a)

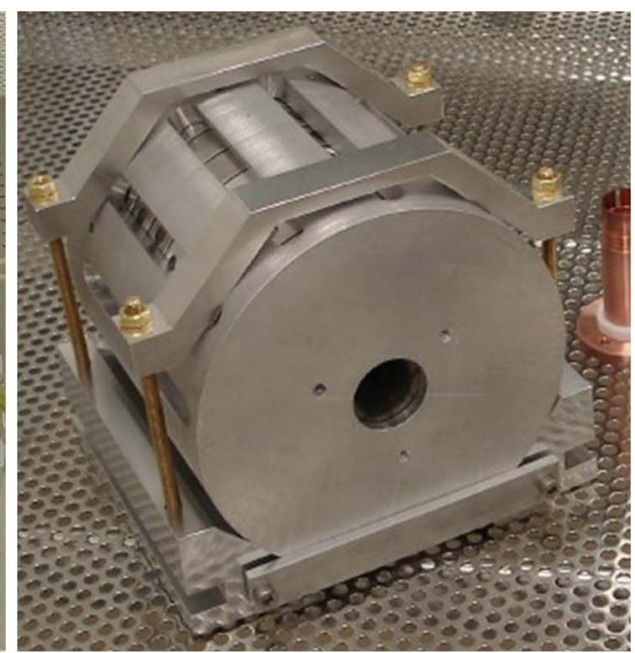

(b)

Fig. 1. (Color online) Faraday rotator magnet system for the (a) LIGO and (b) Virgo VPFI. 


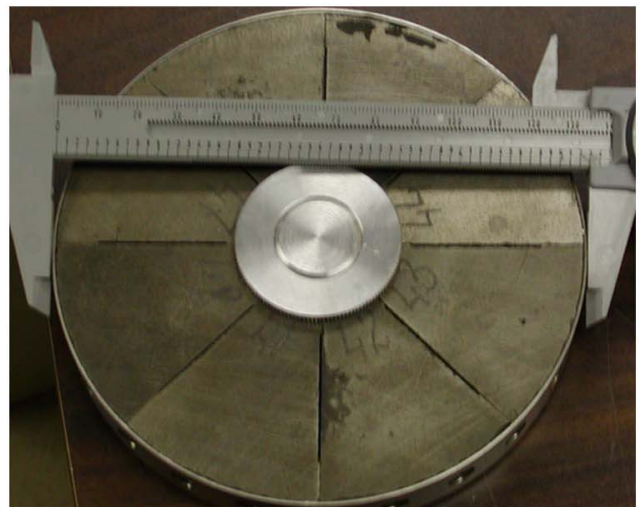

(a)

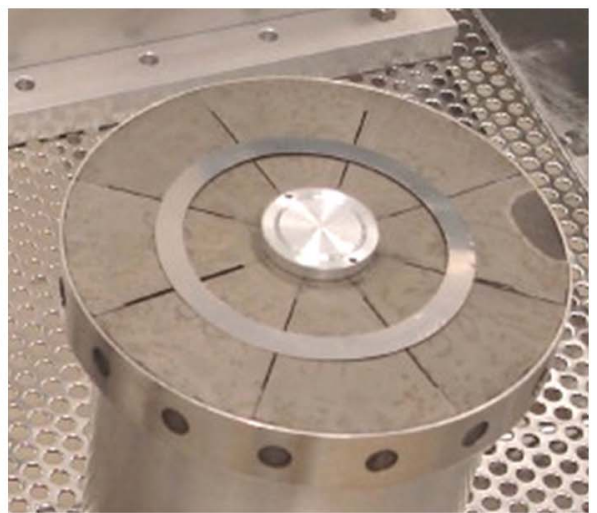

(b)

Fig. 2. (Color online) (a) Individual magnetic ring sectors, showing radial sectors and (b) magnetic system during assembly. The solid aluminum ring located at the center prevents repulsive forces from disassembling the magnetic sectors in the axial direction. The aluminum flat annulus is a shim to allow better outgassing during the vacuum bakeout.

changes in time; dynamic stray magnetic fields arising from low-frequency motion of the Faraday rotator with respect to the mirror couple to the magnets and induce spurious longitudinal and angular motion of the mirrors. Thus, it is critical to have fields and field gradients that die off rapidly away from the rotator body.

A detailed derivation [21] for Advanced LIGO shows that the requirement on the magnetic field to reduce the coupling to suspended mirrors to an acceptable level is given by

$$
\begin{aligned}
\nabla^{2} B_{\|}^{\max }< & 1.35 \cdot 10^{6}\left(\frac{f}{10 \mathrm{~Hz}}\right)^{3 / 2}\left(\frac{3.89 \mathrm{~kg}}{M}\right) \frac{\text { Gauss }}{\mathrm{m}^{2}}, \\
\left(\frac{\partial B_{\perp}}{\partial R}\right)^{\max }= & 3 \cdot 10^{6} \frac{\mathrm{Gauss}}{\mathrm{m}}\left(\frac{f}{\mathrm{~Hz}}\right)^{3 / 2} \\
& \times\left(\frac{8.3 \cdot 10^{-3} \mathrm{~kg} \cdot \mathrm{m}^{2}}{I}\right)\left(\frac{\mu}{0.011 \mathrm{~A} \cdot \mathrm{m}^{2}}\right),
\end{aligned}
$$

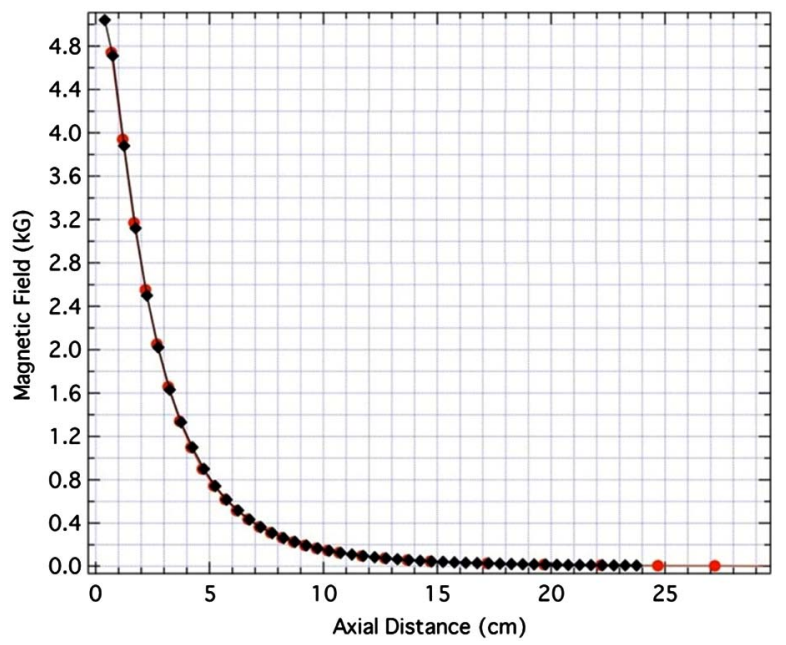

Fig. 3. (Color online) Comparison of the longitudinal magnetic field of a VPFI magnetic disk before (black dots) and after (red dots) baking in a vacuum for $48 \mathrm{~h}$ at a temperature of $60^{\circ} \mathrm{C}$. The fields are identical, demonstrating that vacuum baking under these conditions is not deleterious to the magnetic field. where $B_{\perp, \|}$ are the field components parallel and perpendicular to the magnetic dipole, $M$ is the mass of the mirror, and $I$ is the mirror's moment of inertia about its axis. These calculations are based on the expected low-frequency motion based on the Advanced LIGO seismic isolation design [22].

To determine the field gradients, the magnetic field components were measured using a magnetic probe as a function of distance from the Faraday rotator. The Advanced LIGO and Virgo VPFIs achieve high isolation ratios and compact field designs using structurally similar designs. The difference in the resultant fields in the LIGO Livingston Observatory (LLO) and LIGO Hanford Observatory (LHO) VPFIs is caused by the individual conditions of manufacturing (magnetic alloy properties, the quality of mechanical and thermal processing). In the Virgo VPFI magnetic system, the central axially magnetized ring had bigger outer diameter (90 versus $60 \mathrm{~mm}$ in LIGO VPFIs magnetic system) and higher coercive force of magnetization $(\sim 19 \mathrm{kG})$. As a result, shorter TGG crystals are used in the Virgo VPFI. The axial distribution of the $B_{z}$ inside the Advanced LIGO VPFI is shown in Fig. 4 , while that of the Advanced Virgo is given in Fig. 5. From the Advanced LIGO data, we computed the field gradients outside the Faraday rotator (where the field is monotonically decreasing) by fitting the magnetic field to a function and computing the first and second derivatives. The results are shown in Fig. 6, which displays the axial magnetic field and fit [Fig. 6(a) and the gradients of the field [Fig. 6(b)]. From Fig. 6(b), we determine that the field gradients are sufficiently small within a "stay-clear" zone of $20 \mathrm{~cm}$ from suspended Advanced LIGO mirrors. We tried different fitting curves, and the analysis holds also for the worst case (fit \#3, clearly above the furthest measured points). In any case, the requirement is met at less than $20 \mathrm{~cm}$, which is in the range of measurement (last measured data point is at $18.2 \mathrm{~cm}$ ). The distance to the magnets in measurements illustrated in Fig. 6(a) was limited by the sensitivity of the gaussmeter probe, which is why there are no dots with large distance. The experimental points approximation is based on the LHO measured magnetic field in Fig. 4 (black curve), for data points with $x \geq 10 \mathrm{~cm}$ used in the fitting procedure and apparently it is a $1 /\left(x^{4,5}\right)$ dependency. All specifications of the magnetic field to be in the clear zone are listed in [21]. 


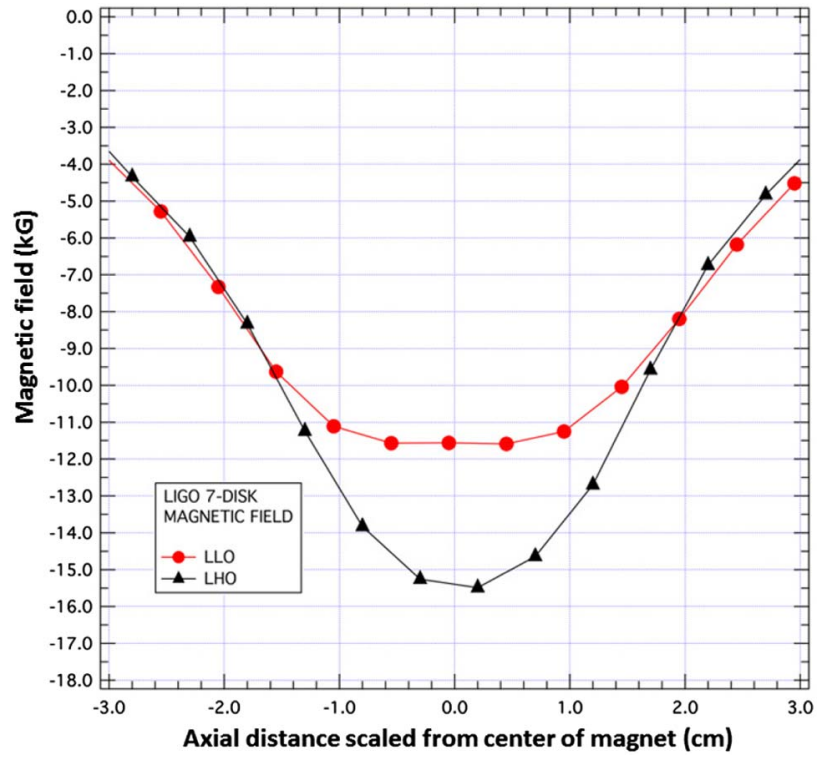

Fig. 4. (Color online) Axial distribution of the magnetic field component $B_{z}$ in the Advanced LIGO VPFI. The graph compares the fields for two different VPFIs, one in operation at the LLO (circles) and one at the LHO (triangles).

\section{Thermally Induced Depolarization and Strategies for} Heat Removal in Vacuum

The primary operational consideration for the MOEs used in the VPFI is the depolarization, $\gamma$, of the radiation in these elements, where $\gamma=P_{\text {orthogonal }} /\left(P_{\text {total }}\right)$ and $P_{\text {orthogonal,total }}$ are the depolarized and incident powers. The depolarization indicates what fraction of the polarization introduced to the VPFI will change its polarization to the orthogonal direction upon backward propagation through the Faraday rotator. "Cold" depolarization occurs in optical elements because of intrinsic imperfections in the magneto-optical medium and nonuniformity of the magnetic field and is typically small $\left(<10^{-4}\right)$. The depolarization caused by laser radiation absorption in the optical elements, the so-called hot or thermally induced depolarization, is dependent on the power of incident optical radiation. At the operational powers of Virgo and LIGO, it greatly exceeds the cold depolarization and determines the isolation ratio at high powers. The isolation ratio, measured in decibels, is given by

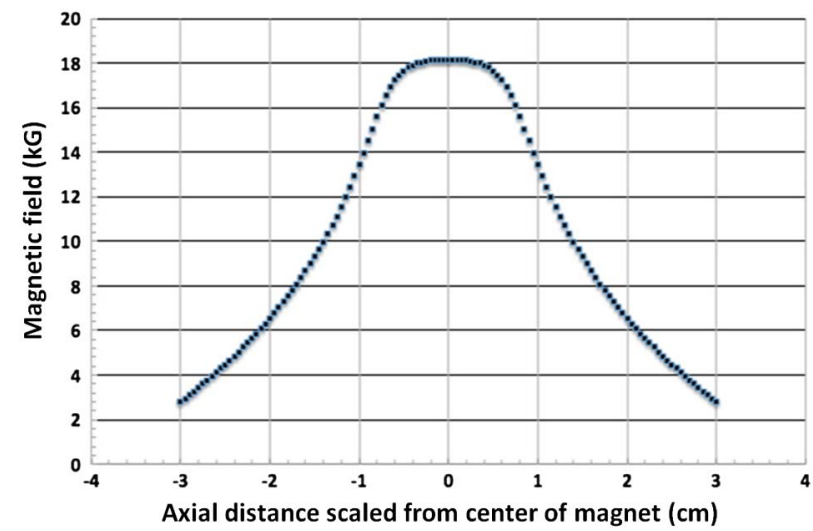

Fig. 5. (Color online) Axial distribution of the magnetic field $B_{z}$ in the Virgo VPFI. The horizontal axis shows axial distance from the center of magnets.

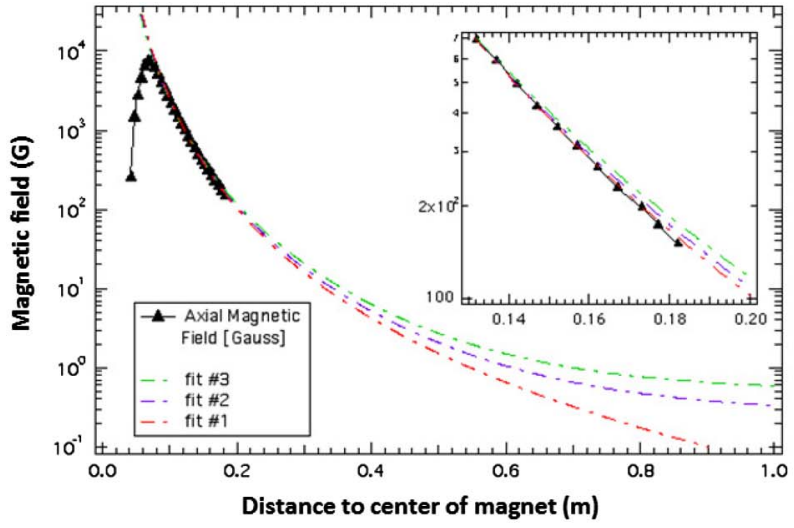

(a)

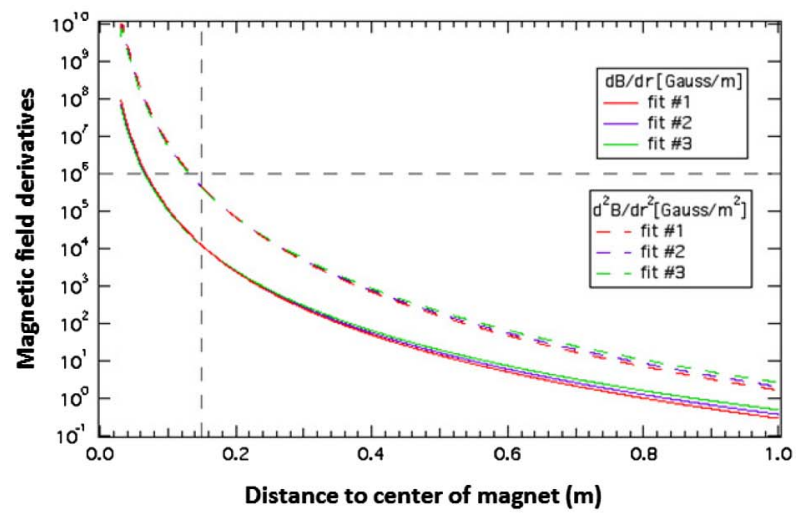

(b)

Fig. 6. (Color online) (a) Axial component of the axial magnetic field of the LIGO VPFI, measured from the center of the rotator, along with fits to three models, discussed in the text. Inset, expanded view of data and fits at the lower measured field values. (b) First (solid lines) and second (dashed lines) derivatives of the magnetic field computed using the fits. The horizontal dashed line shows the $10^{6}$ level. [This value is slightly lower than the requirement for the two derivatives given by Eqs. (2) and (3)]. The vertical dashed line at $15 \mathrm{~cm}$ shows that for larger distances, the field derivatives are below the critical values for noise coupling to the suspended mirrors.

$$
I[\mathrm{~dB}]=-10 \cdot \log (\gamma) .
$$

Thermally induced depolarization in TGG depends on the crystal orientation with respect to the incident radiation polarization [23]. Figure 7 shows the results of measurements of thermally induced depolarization for [001] TGG crystals such as those used in Virgo, oriented to produce the maximum thermally induced depolarization. The data points are experimentally measured values for individual crystals (squares, circles) and both crystals in series (triangles). Depolarization in two TGGs is equal to quadruple depolarization in a single TGG, in exact accordance with the theory. The solid lines correspond to a theoretical calculation for thermally induced depolarization [23]. Figure 7 shows that while both crystals have rather good optical quality, in order to limit the backpropagating light to less than $10^{-3}(30 \mathrm{~dB})$ required in gravitational wave detector operation [24] at the level of its operating laser power $[\underline{2}, \underline{5}, \underline{6}]$, compensation of the thermally induced depolarization using an intermediate quartz rotator is needed for the VPFI design [8]. 


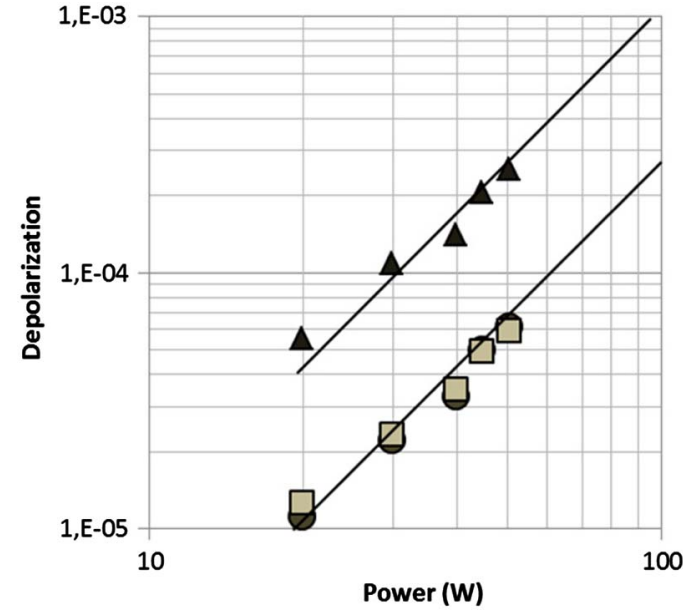

Fig. 7. (Color online) Experimentally measured depolarization (symbols) and theoretical predictions (lines) for the dependence of the depolarization on laser power for the Virgo VPFI. The squares and circles are the measured depolarizations for each crystal in the VPFI The triangles display the measurement for both crystals in series. Good agreement with theory is seen.

To enhance the performance of the VPFI in vacuum, additional considerations come into play. Convective cooling of the TGG crystal is suppressed in vacuum, resulting in a reduction in optical isolation with respect to its in air performance (as we show in Subsection 3.A below). To improve heat conduction and limit thermo-optical degradation, specially designed mounts are used for the rotator elements that provide improved heat conduction (Fig. 8). The TGG crystal barrels are wrapped in $0.15 \mathrm{~mm}$ thick indium foil and mounted in tapered collets with end caps that compress the indium and provide more uniform contact of the TGG to the crystal holder. The holder is directly mounted to the Faraday rotator housing through multiple contact points, permitting an improved heat conduction path.

\section{EXPERIMENTAL RESULTS}

\section{A. LIGO}

The VPFI design was subjected to a comprehensive experimental test program to verify that it meets the requirements for Advanced LIGO. We first investigated the performance of the FI in air using the following two configurations: a hybrid configuration consisting of a fused silica thin film polarizer (TFP) and a calcite wedge polarizer (CWP), which is optimal for the reflected beam pickoff (which was used in previous LIGO installation when directing of the reflected beam to a particular port was needed and CWP was not usable), and a pair of identical CWPs, which is more suitable for high isolation. The optical isolation was determined according to Eq. (4). In each case, the setup was optimized for maximum isolation at the highest power $(103 \mathrm{~W})$ and not adjusted thereafter. In Fig. 9, the isolation ratio versus incident optical power is displayed.

The TFP + CWP configuration presents a lower isolation ratio $(39 \mathrm{~dB})$, limited by the extinction ratios of the TFPs (measured to be $40 \mathrm{~dB}$ ). An isolation ratio of $49 \mathrm{~dB}$ is obtained at $103 \mathrm{~W}$ for CWP + CWP, essentially constant for power changes from 80 to $103 \mathrm{~W}$; however it decays to $44 \mathrm{~dB}$ due to a small reduction in the temperature of the TGG as the power is reduced.

Temperature changes are likely to be larger in vacuum; thus we tested the VPFI at various input powers as a function of pressure to determine how the isolation ratio degraded. For these tests, a standard TGG and quartz crystal holder was used rather than the more efficient holders described in Subsection 2.C above. The isolation ratio was optimized at ambient atmospheric pressure and then monitored as the pressure was lowered. We observe a strong degradation as the pressure drops, attributable to the less efficient convective cooling of the absorbing optical elements of the VPFI. The isolation degradation at lower pressure values is shown in Fig. 10.

To determine the level the isolation ratio can be restored in vacuo, a vacuum-compatible motorized rotation stage was used in the chamber to control the half-wave plate. In Fig. 11(a), a VPFI configuration with the two calcite polarizers and without thermal lens compensator deuterated potassium dihydrogen phosphate (DKDP) crystal was exposed for $1 \mathrm{~h}$ followed by adjustment of the wave plate for best isolation. A vacuum pressure of $6 \cdot 10^{-5}$ torr and $30 \mathrm{~W}$ laser power was used in this experiment. The isolation was then measured at $10 \mathrm{~min}$ intervals during the next $90 \mathrm{~min}$, showing a decrease from $40 \mathrm{~dB}$ to just below $36 \mathrm{~dB}$ with an exponential decrease [ $\tau=74 \mathrm{~min}$, dashed line in Fig. 11(a)]. At $130 \mathrm{~min}$ after initial exposure, the isolation can be improved from 36 to $40 \mathrm{~dB}$ by rotating the wave plate by approximately $1^{\circ}$. However the isolation ratio continues to degrade because the adjustment took place before thermal equilibrium had been reached.

This latter effect is shown clearly in Fig. 11(b). The experiment was repeated with the DKDP placed in between the two

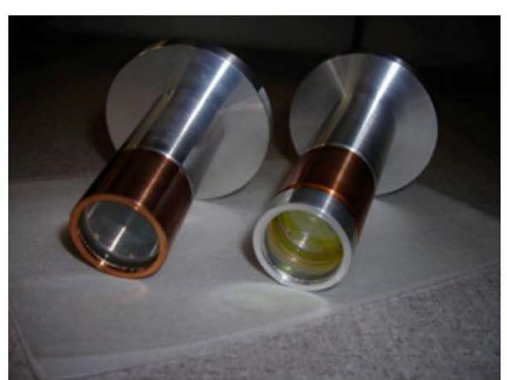

(a)

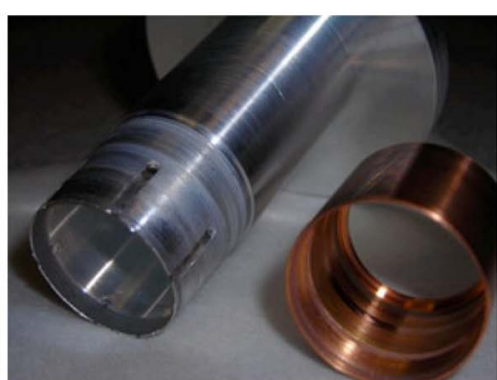

(b)

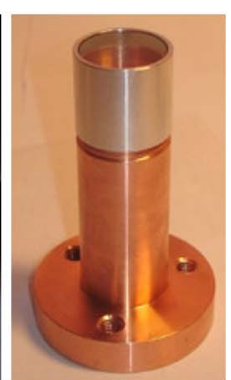

(c)

Fig. 8. (Color online) TGG holders designed for more efficient heat transfer by conduction: (a) holders for LIGO TGG crystal (left) and quartz/TGG (right), (b) an open TGG holder showing the tapered collet and copper cap, and (c) the Virgo TGG holder design, which uses copper instead of aluminum as the main body. Copper is almost twice as thermally conductive as aluminum. 


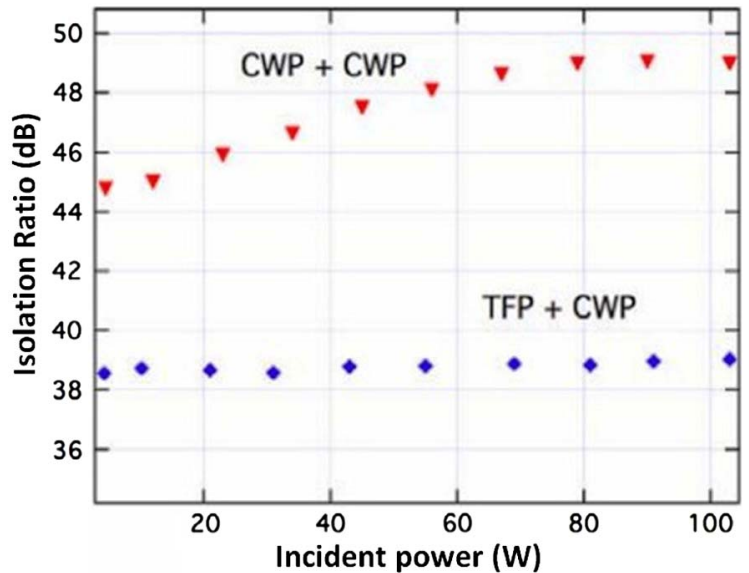

Fig. 9. (Color online) Power-dependent optical isolation for VPFI using one TFP and CWP (blue diamonds) and a pair of CWPs (red triangles).

calcite polarizers and the isolation was recovered again with the motorized half-wave plate [Fig. 11(b)]. The wave plate was adjusted at $120 \mathrm{~min}$ to recover the isolation to $36 \mathrm{~dB}$. The isolation was stable after $12 \mathrm{~h}$, with the isolation ratio improving to $43 \mathrm{~dB}$, as the wave plate overcompensated for the loss of isolation, with the subsequent temperature increase producing a more fine adjustment of the polarization rotation. The heat sink from the crystals was improved by using special holders and indium foil for wrapping the crystals, which allow a significant decrease in the time period before thermal equilibrium had been reached. For Advanced LIGO, a motorized half-wave plate will be inserted to restore the isolation. In addition, for Advanced LIGO VPFI CWP + CWP configuration will be used. The forward transmission for the Advanced LIGO VPFI is $\sim 97 \%$, but the thermal lensing makes an effective transmission around $93 \%$.

We also measured the deflection of the rejected backpropagating beam for the VPFI. Experience with commercial FIs in initial LIGO showed that the backpropagating rejected beam undergoes large changes in pointing ("thermal beam steering") as the laser power is changed. To assess these effects, we measured how the propagation direction of the

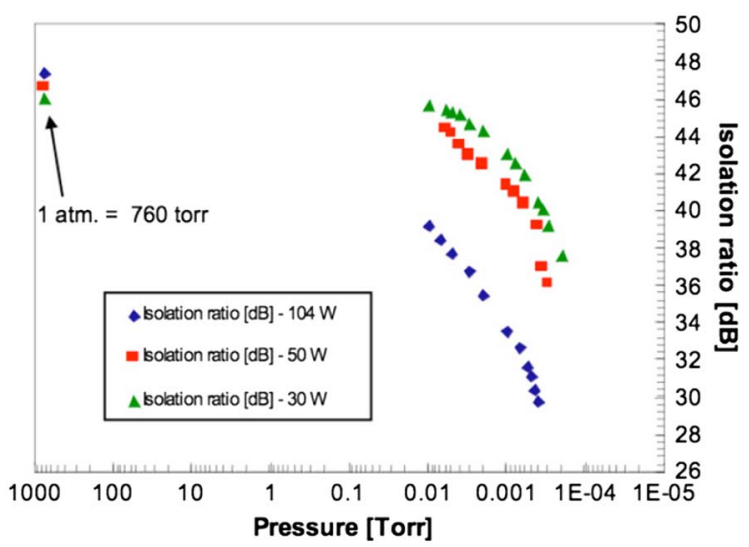

Fig. 10. (Color online) Isolation ratio degradation as a function of pressure for input laser powers of 104,50 , and $30 \mathrm{~W}$, respectively. At all powers, a substantial reduction in the isolation ratio is observed, with the most severe reduction at $104 \mathrm{~W}$. rejected beam was affected by thermal loading of the VPFI. Measurements were made using $30 \mathrm{~W}$ laser power, and the beam position was recorded as a function of time on a quadrant photodiode. Zero time corresponds to the time at which the power was increased from 0 to $30 \mathrm{~W}$. Measurements were made on two different configurations-a VPFI using CWPs and one using TFPs.

The thermal steering of the VPFI using CWPs is shown in Fig. 12(a). The beam drift was measured after blocking/unblocking the beam in front of the FI. This way the rest of the system beside VPFI is thermalized, and the drift is caused only by the FI. The different slopes are various components/ crystals/optics with different thermal conductivities and heat loss depending on how they were mounted. It took 1000$2000 \mathrm{~s}$ to reach stable operation. The nearly flat data from $3000-4500 \mathrm{~s}$ is probably a measure of overall beam stability. Measurements taken at lower powers (not shown) show that the magnitude of the drift is linear in power. The vertical and horizontal drifts are comparable in magnitude, and a thermal steering coefficient of $1.3 \mu \mathrm{rad} / \mathrm{W}$ is found from the data.

Thermal steering using TFPs is shown in Fig. 12(b), corresponding to a thermal steering coefficient of $130 \mathrm{nrad} / \mathrm{W}, \mathrm{a}$ factor of 10 improvement. The vertical deflection is slightly larger than the horizontal deflection. This improvement is likely due to a combination lower absorption in the fused silica TFPs and also asymmetric heating of the CWPs, which by their geometry are thicker on one side of the beam.

Thermal lensing was determined by measuring the beam divergence in air as a function of incident laser power for (i) the VPFI alone (without the compensating DKDP crystal), (ii) the DKDP alone, and (iii) the entire thermally compensated VPFI. In this case, the DKDP was placed before the FI and only the single pass lensing was measured. Figure 13 displays the thermal lensing induced in the VPFI as a function of single pass power. For these measurements, there was no backward propagation. The total thermal lensing (green triangles) at $30 \mathrm{~W}$ of incident power is negligible; at $70 \mathrm{~W}$, a focal power of approximately $-0.025 \mathrm{~m}^{-1}$ (corresponding to a thermal focal length $f=-40 \mathrm{~m}$ ). The magnitude of thermal compensation depends on the absorption coefficient and the length of the DKDP crystal. It is difficult to control the absolute absorption in DKDP since it depends sensitively on the stoichiometry of deuterium.

For these measurements, it was known in advance from absorption measurements that the DKDP would overcompensate for the thermal lens in the FR. Better results can be obtained by further thinning of the DKDP crystal; the DKDP will be optimized for the FIs used in the advanced LIGO interferometers.

\section{B. Virgo}

The VPFI has some differences with respect to the LIGO VFPI, including the magnetic field configuration and the higher thermal conductivity TGG holders. Moreover, the assembled magnetic system has been baked for about one week at $50 \mathrm{C}^{\circ}$, followed by a one month period under vacuum ( $10^{-6}$ torr). Following the bake and vacuum treatment, an isolation ratio (at low laser power) was measured to be $43 \mathrm{~dB}$. The forward transmission was measured to be $95 \%$, consistent with the expected value based on transmissions of the individual elements. 

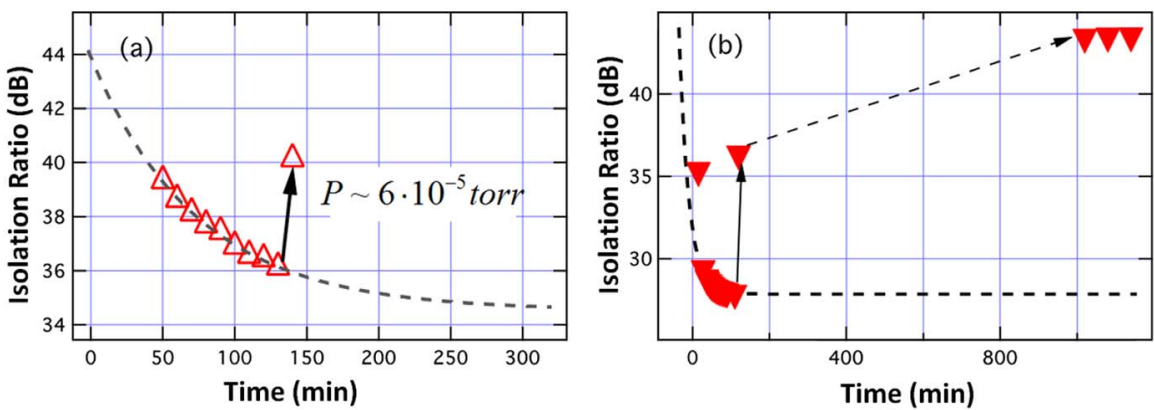

Fig. 11. (Color online) (a) Isolation degradation in vacuum and restoration by adjusting a motorized half-wave plate in situ. Loss of isolation ratio versus time for an FI without thermal lens compensation. Time $t=0$ corresponds to the turn on of the laser. The dashed line is a decaying exponential fit with a $74 \mathrm{~min}$ time constant. At $130 \mathrm{~min}$, the wave plate is adjusted. (b) Similar plot showing isolation reduction and restoration for an FI incorporating DKDP thermal lens compensation. The improvement at long times is a consequence of controlled overcompensation.

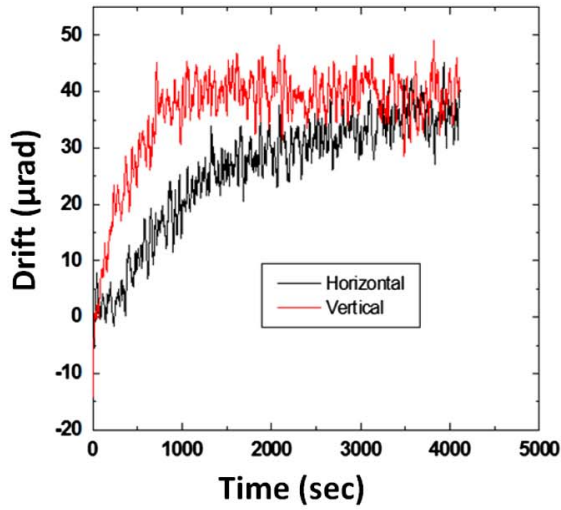

(a)

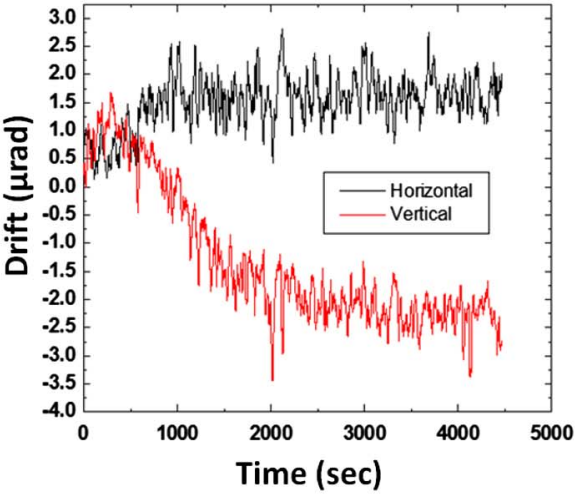

(b)

Fig. 12. (Color online) (a) Change in angle of the rejected FI beam versus time for calcite polarizers from a cold start (no power) to $30 \mathrm{~W}$. (b) Change in angle of the rejected FI beam versus time for TFPs from a cold start (no power) to $30 \mathrm{~W}$.

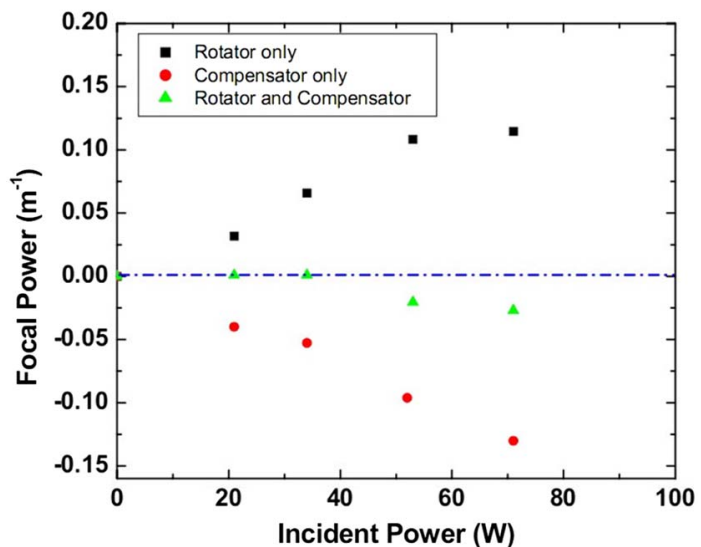

Fig. 13. (Color online) Thermal focal power of the FI as a function of incident laser power. The black squares show the focal power for the FI without thermal compensation. The red circles display the measured focal power of the DKDP. The green triangles display the measured focal power for the fully compensated FI.

The VPFI was installed in the vacuum chamber at a residual pressure of the order of $\sim 2 \cdot 10^{-6}$ torr. Figure 14 shows the comparison of in-air and in-vacuum isolation ratios versus the laser power. For these measurements, the laser was double-passed through the VPFI. For each power setting, the remotely controllable half-wave plate was adjusted to minimize the effect of thermal variation of Verdet constant

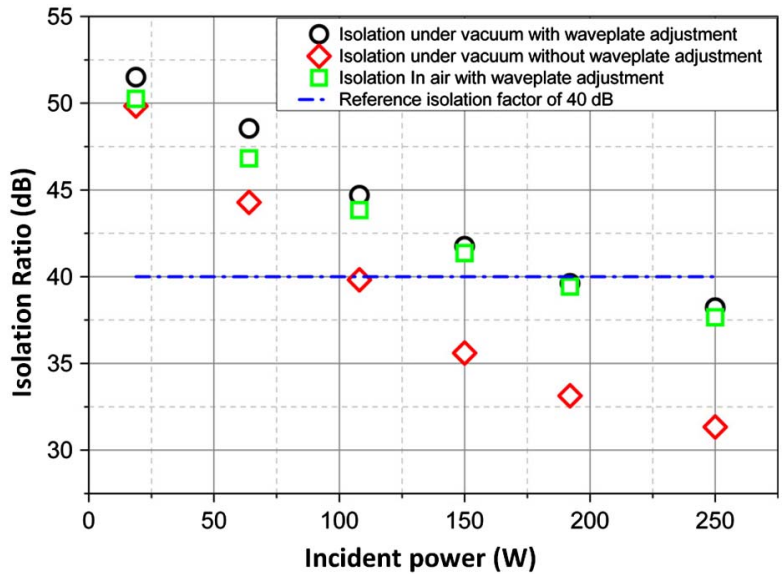

Fig. 14. (Color online) Experimental dependence of isolation ratio versus laser power in vacuum (circles) and in air (squares) with half-wave plate readjustment. The solid line shows desired level for Virgo isolation of $40 \mathrm{~dB}$. The third experimental curve (diamonds) gives the FI the best isolation when there is no readjustment of the half-wave plate to compensate for Verdet constant change.

$[15,25]$ and therefore gets the optimum isolation. As shown in Fig. 14, with $250 \mathrm{~W}$ traversing the VPFI, the isolation ratio is $36.5 \mathrm{~dB}$. The isolation ratio is sensitive to alignment; in particularly good alignment conditions, $38 \mathrm{~dB}$ was achievable. A comparison of in-air and in-vacuum performances of the VPFI shows that the measurements are very close, indicating that 


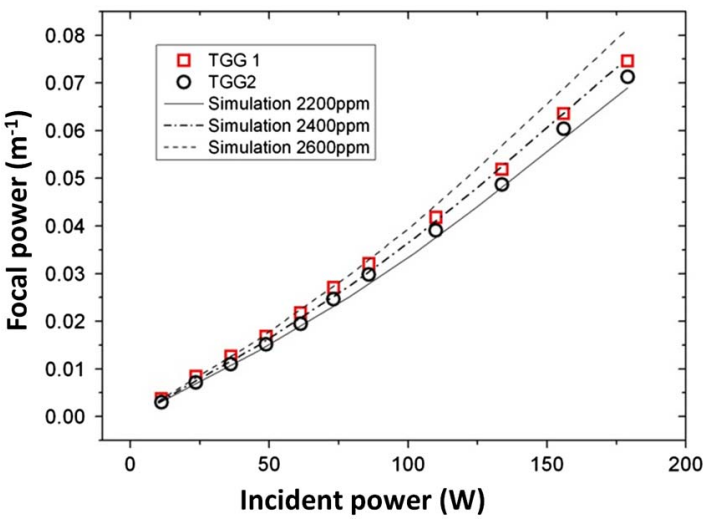

Fig. 15. (Color online) Measurements of thermal lensing in Virgo TGG crystals using a pump-probe technique. The data points are measurements of two individual TGG crystals. The lines are estimated absorption in the TGG crystals based on a finite element model described in the text.

we are limited only by residual thermal depolarization, which is expected to be the same in air or in vacuum.

The in-vacuum performances of the FI were also compared with and without compensation of the Verdet constant change with the half-wave plate. The isolation drop is about $6-7 \mathrm{~dB}$ at $250 \mathrm{~W}$ (Fig. 14). This is quite limited and is consistent with the limited temperature increase. We attribute the limited change in isolation ratio to the copper holders, which are very efficient in removing heat from the TGG crystals.

The thermal lensing effect in the VPFI was evaluated using a pump-probe measurement by looking at the modification of a probe beam of a low-power single mode laser (JDS Uniphase $100 \mathrm{~mW}$ NPRO laser) in the TGG crystals heated by the $200 \mathrm{~W}$ pump laser from IPG Photonics (IPG YLR-200-LP). In these experiments, no compensation of the thermal lens was used. The probe beam was sent through a single TGG crystal in the opposite direction and polarization with respect to the pump beam. The probe laser was then analyzed with a Shack-Hartmann wavefront sensor. The use of a pump/probe setup enables the realization of a differential measurement on the probe beam with the pump laser on/off. With this method, it is then possible to sense even very small thermal lenses (up to $1000 \mathrm{~m}$ ). Figure 15 gives the results of thermal lens focal power measurements for a laser power going from 10 to $180 \mathrm{~W}$ for the two different TGG crystals (TGG1 and TGG2) used in the VPFI.

To simulate the effect of heat in a FI, we have developed a finite element modeling (FEM) simulation using MATLAB. This model computes the absorbed energy from a heating Gaussian beam and makes a map of temperature using the thermal conduction law inside and the Stefan-Boltzmann law at the limits of the heated crystal. As convection has a nonnegligible effect, we use an empirical model of convection described in [26]. The FEM simulation gives as output a temperature map of the crystal from which we extract its refractive index map using the TGG thermo-optic coefficient. This enables us to calculate the induced thermal lensing effect. Using this simulation we are able to estimate the linear absorption of each TGG rod as shown in Fig. 15. From this measurement, we found absorption levels of $2300 \mathrm{ppm} / \mathrm{cm}$ $+/-100 \mathrm{ppm} / \mathrm{cm}$ for TGG1 and $2600 \mathrm{ppm} / \mathrm{cm}+/$ $-100 \mathrm{ppm} / \mathrm{cm}$ for TGG2.

\section{CONCLUSION}

We have presented a comprehensive investigation of highpower FIs designed for future high-power gravitational-wave interferometers. Innovations in the design of the magnet system and the mechanical components lead to reductions in isolation loss, thermal lensing, and thermally induced beam steering. Although the LIGO and Virgo VPFIs are somewhat different in design, our investigations indicate that both will perform well in Advanced LIGO and Advanced Virgo.

\section{ACKNOWLEDGMENTS}

This work was supported by the National Science Foundation (NSF) through grants PHY-0757968, PHY-0855313, and PHY0555453 and by the European Gravitational Observatory through a grant for the development of "High power optical component for Advanced Virgo." This document has been assigned LIGO doc. no. P1100095.

\section{REFERENCES}

1. LIGO Science Collaboration, "LIGO: the Laser Interferometer Gravitational-Wave Observatory,” Rep. Prog. Phys. 72, 076901 (2009).

2. Virgo Collaboration, "Status of the Virgo project," Class. Quantum Grav. 28, 144002 (2011).

3. H. Grote (for the LIGO Scientific Collaboration), "The GEO 600 status," Class. Quantum Grav. 27, 084003 (2010).

4. TAMA Collaboration, "Current status of TAMA," Class. Quantum Grav. 19, 1409-1419 (2002).

5. G. M. Harry, "Advanced LIGO: the next generation of gravitational wave detectors," Class. Quantum Grav. 27, 084006 (2010).

6. LCGT Collaboration, "Status of LCGT," Class. Quantum Grav. 27, 084004 (2010).

7. D. H. Reitze, "Faraday isolator specifications for advanced LIGO,” LIGO-T050226-00-D (2006).

8. E. A. Khazanov, N. F. Andreev, A. A. Babin, A. Kiselev, O. V. Palashov, and D. H. Reitze, "Suppression of self-induced depolarization of high-power laser radiation in glass-based Faraday isolators," J. Opt. Soc. Am. B 17, 99-102 (2000).

9. G. Mueller, R. Amin, D. Guagliardo, Donavan McFeron, R. Lundock, D. H. Reitze, and D. B. Tanner, "Method for compensation of thermally induced modal distortions in the input optics components of gravitational wave interferometers," Class. Quantum Grav. 19, 1793-1801 (2002).

10. E. A. Khazanov, N. F. Andreev, A. N. Mal'shakov, O. V. Palashov, A. K. Poteomkin, A. M. Sergeev, A. A. Shaykin, V. V. Zelenogorsky, I. Ivanov, R. Amin, G. Mueller, D. B. Tanner, and D. H. Reitze, "Compensation of thermally induced modal distortions in Faraday isolators," IEEE J. Quantum Electron. 40, 1500-1510 (2004).

11. D. Coyne, LIGO vacuum compatible materials list, LIGOE960050-B-E (Laser Interferometer Gravitational Wave Observatory, 2004).

12. D. S. Zheleznov, E. A. Khazanov, I. B. Mukhin, O. V. Palashov, and A. V. Voytovich, "Faraday rotators with short magnetooptical elements for $50 \mathrm{~kW}$ laser power," IEEE J. Quantum Electron. 43, 451-457 (2007).

13. L. Williams and V. Quetschke, aLIGO Faraday rotator magnet assembly hazard analysis, E070201-00-D LIGO-E1000110-v1 (2010).

14. Bao-Min Ma and K. S. V. L. Narasimhan, "Temperature dependence of magnetic properties of Nd-Fe-B magnets," J. Magn. Magn. Mater. 54-57, 559-562 (1986).

15. N. P. Barnes and L. P. Petway, "Variation of the Verdet constant with temperature of terbium gallium garnet," J. Opt. Soc. Am. B 9, 1912-1915 (1992).

16. E. A. Khazanov, O. V. Kulagin, S. Yoshida, D. Tanner, and D. Reitze, "Investigation of self-induced depolarization of laser radiation in terbium gallium garnet," IEEE J. Quantum Electron. 35, 1116-1122 (1999). 
17. E. A. Khazanov, N. F. Andreev, O. V. Palashov, A. K. Poteomkin, A. M. Sergeev, O. Mehl, and D. H. Reitze, "Effect of terbium gallium garnet crystal orientation on the isolation ratio of a Faraday isolator at high average power," Appl. Opt. 41, 483-492 (2002).

18. I. B. Mukhin, O. V. Palashov, and E. A. Khazanov, "Reduction of thermally induced depolarization of laser radiation in [110] oriented cubic crystals," Opt. Express 17, 5496-5500 (2009).

19. I. B. Mukhin, O. V. Palashov, E. A. Khazanov, A. Ikesue, and Y. L. Aung, "Experimental study of thermally induced depolarization in Nd:YAG ceramics," Opt. Express 13, 5983-5987 (2005).

20. M. V. Plissi, K. A. Strain, C. I. Torrie, N. A. Robertson, S. Killbourn, S. Rowan, S. M. Twyford, H. Ward, K. D. Skeldon, and J. Hough, "Aspects of the suspension system for GEO 600,” Rev. Sci. Instrum. 69, 3055-3061 (1998).
21. G. Mueller, S. Stepuk, and V. Quetschke, Analysis of stray magnetic fields from the Advanced LIGO Faraday Isolator, LIGO-T060025-00-D (2006).

22. LIGO Collaboration, "Seismic isolation requirements for advanced LIGO," Class. Quantum Grav. 19, 1591-1597 (2002).

23. E. A. Khazanov, "Compensation of thermally induced polarization distortions in Faraday isolators," Quantum Electron. 29, 59-64 (1999).

24. M. A. Arain, A. Lucianetti, R. Martin, G. Mueller, V. Quetschke, D. H. Reitze, D. B. Tanner, L. Williams, and W. Wu, Advanced LIGO input optics subsystem preliminary design document, LIGO-T060269-02-D (2007).

25. Virgo Collaboration, "In-vacuum Faraday isolation remote tuning," Appl. Opt. 49, 4780-4790 (2010)

26. Virgo Collaboration, "In-vacuum optical isolation changes by heating in a Faraday isolator," Appl. Opt. 47, 5853-5861 (2008). 